\title{
Um modelo de monitoramento de pacientes utilizando a ciência de situação
}

\author{
Lucas Closs, Cristiano André da Costa, Rodrigo da Rosa Righi \\ Programa de Pós-Graduação em computação Aplicada \\ Universidade do Vale do Rio dos Sinos (UNISINOS) \\ Avenida Unisinos, 950 - 93022-750 - São Leopoldo - RS - Brasil \\ lucas.closs@gmail.com, \{cac, rrrighi\}@unisinos.br
}

\begin{abstract}
The constant advances in mobile and ubiquitous computing enable new developments and features in several areas, health being one of them. From this, arise the concept of ubiquitous care, where new ways of medical area apllied like patient monitoring. In view of this feature, the SAC Situation-Aware Care model proposes the use of patient monitoring together with situation awareness to perceive, comprehend and project deviations and degradations in the health of patients, providing the best medical information to doctors. To assess the viability of the SAC model was applied an evaluation by scenario, which is supported by the development of a prototype.
\end{abstract}

Resumo. Os constantes avanços na computação móvel e ubíqua possibilitam novos desenvolvimentos e recursos em diversas áreas, sendo a saúde uma destas. A partir disto, surge então o conceito de cuidados ubíquos, onde são aplicadas novas formas cuidados médicos, como o monitoramento de pacientes. Tendo em vista este recurso, o modelo SAC - Situation-Aware Care propõe o uso do monitoramento de pacientes em conjunto da ciência de situação para perceber, compreender e projetar desvios e degradações na saúde, disponibilizando aos médicos melhores informações para atendimento. Para avaliar a viabilidade do modelo SAC foi aplicado um estudo de caso, baseado em cenário, sendo este apoiado pelo desenvolvimento de um protótipo do modelo.

\section{Introdução}

A comercialização de dispositivos móveis (Mobile devices), principalmente de smartphones, segundo a Gartner, no ano de 2015 foram comercializadas um total de 1.917 bilhões de telefones móveis, onde os smartphones representam $70 \%$ do total [MEULEN; WOODS, 2016]. Para o ano de 2016, a Gartner projeta um aumento de $1,4 \%$ no comércio de telefones móveis - 1.943 bilhões unidades - e também um aumento de 7\% na representação de smartphones - atingindo 77\% [MEULEN; WOODS, 2016]. Tal circunstância, acaba por incentivar e influenciar no desenvolvimento e nos estudos de novas tecnologias e novos recursos para a área de Computação Móvel (Mobile Computing). Outro nicho de dispositivos que vem se destacando tanto para os consumidores quanto para a comunidade acadêmica são os dispositivos vestíveis (Wearable devices) [PATEL; ASCH; VOLPP, 2015]. Estes, são capazes de coletar diversos dados de seu utilizador. Indo além desta avaliação individual 
dos conceitos acima destacados, a aplicação combinada dos mesmos disponibiliza novos recursos para a Computação Ubíqua (Ubiquitous Computing), onde temos a tecnologia fortemente integrada ao cotidiano da sociedade [WEISER, 1991] [FRIEDEWALD; RAABE, 2011].

Conforme mencionado, os novos desenvolvimentos e os novos recursos disponíveis para a computação móvel e ubíqua, estão permitindo a manifestação de oportunidades em diferentes áreas de aplicação, como: saúde, educação, comércio e transportes [FELTES; BARBOSA, 2014]. Na área da saúde, destaca-se o segmento de Cuidados Ubíquos que intentam prover melhores condições, informações e recursos aos hospitais e aos médicos, tendo como consequência a melhora no atendimento e no tratamento dos pacientes [GELOGO; KIM, 2013]. Um destes recursos disponibilizados seria o monitoramento de pacientes, que tem como objetivo o acompanhamento diário do paciente registrando os seus sinais vitais. Entretanto, este recurso pode ser ampliado com a aplicação de uma camada de inteligência. Essa camada atua sobre estes dados detectando possíveis desvios ou problemas relacionados com a saúde do paciente. Tendo em vista esta lacuna, esse trabalho propõe o emprego da Ciência de Situação (Situation Awareness) [ENDSLEY, 1995] sobre estes dados transformando-os em informações que representam a situação atual do paciente e a projeção de futuros estados.

O modelo SAC - Situation-Aware Care tem como foco a implementação dos três níveis da ciência de situação, onde apresenta projeções de futuros estados como resultado e não somente compreensões sobre a situação atual, projetando assim possíveis desvios, degradações ou problemas na saúde deste paciente. Tais inferências permitem controles de registros médicos mais apurados sobre as condições do paciente, melhores diagnósticos e principalmente o monitoramento do paciente. Sendo está uma das principais contribuições do modelo SAC. Para este artigo foram avaliados outros cinco modelos, que também utilizam a ciência de situação, mas com temáticas distintas. Porém, dentre os cinco modelos avaliados, somente dois deles tiveram a aplicação das três fases da ciência de situação. Outro aspecto não abordado pelos trabalhos relacionados considerados é quanto a padronização dos dados e das informações processadas pelos mesmos. A padronização garante uma melhor estruturação assim como a interoperabilidade com aplicações terceiras e até mesmo com instituições médicas. $\mathrm{O}$ modelo SAC irá utilizar as definições propostas pelo openEHR [GONZÁLEZ-FERRER, 2015].

O artigo apresenta seis seções. A segunda seção fundamenta sobre os principais conceitos utilizados. A terceira seção apresenta as análises sobre os trabalhos relacioandos. Na quarta seção é descrito o modelo SAC. A quinta seção descreve sobre a avaliação realizada. E por fim, apresenta a conclusão e os futuros trabalhos.

\section{Cuidados Ubíquos e Ciência de Situação}

Cuidados ubíquos surgem com a intenção aumentar a eficiência, a acuracidade e disponibilidade de tratamentos médicos [GELOGO; KIM, 2013]. Uma das abordagens de cuidados ubíquos seria o monitoramento de pacientes, que possibilita que hospitais e médicos tenham conhecimento sobre sinais vitais de seus pacientes no decorrer do dia [GELOGO; KIM, 2013]. Tal conhecimento permite: um melhor monitoramento sobre 
os tratamentos médicos ou estado do paciente; e diagnósticos mais acurados [GELOGO; KIM, 2013]. Os cuidados ubíquos, além de disponibilizarem mais dados e informações, possibilitam que o próprio paciente tenha melhor conhecimento sobre a sua saúde, verificando variações e degradações em seus sinais vitais, servindo assim como um indicador, para que o mesmo tenha maiores e melhores cuidados com a sua saúde.

A ciência da situação, originalmente utilizada pelo setor da aviação, foi definida por Endsley (1995) como a percepção dos elementos de um ambiente dentro de um período de tempo, como a compreensão sobre o significado dos mesmos e assim como a projeção de seus estados em um futuro próximo. Endsley (2015) descreve o processo de análise da situação possuindo três fases. A primeira fase, atua na percepção e reconhecimento de atributos e estados de elementos relevantes do ambiente (localização, altitude e destino próprio e de outras aeronaves). Na segunda fase, compreensão sobre a situação, tem-se a síntese dos elementos percebidos pela primeira (tempo e distância a ser percorrida com o combustível disponível). E a terceira fase, baseia-se na habilidade de projetar ações e prever estados futuros dos elementos do ambiente (projeção de desvios no cronograma; projeção da trajetória própria e de outras aeronaves). Estas projeções e predições são recursos valiosos nas tomadas de decisões. A ciência de situação vem sendo aplicada em diversas áreas da saúde, tais como: monitoramento de pacientes com problemas cardiológicos [DA ROCHA; DA ROSA; RIGHI, 2015], monitoramento de pacientes anestesiados [FIORATOU et al., 2010], apoio a decisão em diagnósticos de pacientes [SINGH et al., 2012], dentre outras.

\section{Trabalhos Relacionados}

Foram avaliados cinco modelos que utilizam o conceito de monitoramento de pacientes e ciência de situação. O artigo de Don et al. (2013) apresenta uma proposta de modelo para realizar o monitoramento de pacientes remotamente, tendo como foco a utilização da ciência da situação. $\mathrm{O}$ modelo toma decisões de modo declarativa, ajudando a equipe médica a compreender a situação.

O trabalho de Delir Haghighi et al. (2014) propõe uma modelo de monitoramento móvel da saúde utilizando ciência de situação. $\mathrm{O}$ modelo proposto integra o conhecimento de alto nível com os dados sensoriais de baixo nível, utilizando como mecanismo de raciocínio, uma adaptação da Lógica Fuzzy, chamada pelos autores de Inferência Fuzzy de Situação (Fuzzy Situation Inference).

O artigo escrito por Wai et al. (2010) apresenta um modelo que utiliza o sensoriamento do ambiente aliado a ciência de situação para monitorar possíveis eventos perigosos, quando pacientes estão deitados ou ao redor da cama ao redor. Para isso, são utilizados sensores de pressão e vídeo. O modelo aplica o conceito de sistema especialista, para realizar as inferências sobre o paciente. A proposta principal do modelo é permitir um monitoramento sobre o bem-estar dos pacientes.

Os autores Rocha, Costa e Righi (2015) apresentam o modelo UbHeart, que tem como proposta o monitoramento de pacientes com insuficiência cardíaca utilizando a ciência de situação para identificar possíveis riscos à saúde do paciente. A recorrência desta patologia, acaba por diminuir a qualidade de vida do paciente assim como influência em maiores gastos para o sistema de saúde, sendo estes alguns dos motivos pela definição da temática do artigo. 
O trabalho de Pereira, Costa e Almeida (2013) apresenta um modelo de plataforma para gerenciamento de situação. Plataforma chamada de SCENE, permite a especificação de regras baseadas em situação. $\mathrm{O}$ modelo proposto, de acordo com o conceito de ciência de situação de Endsley (1995), encontra-se no nível de Avaliação da Situação, no qual engloba a percepção e a compreensão sobre a situação.

A ciência da situação, conforme apresentado anteriormente, possui três fases: percepção, compreensão e projeção. Nos trabalhos estudados, foi indicado pelos autores a aplicação deste conceito, porém, ao realizar a análise os modelos propostos, se identificou que o terceiro nível da ciência de situação - projeção de futuros estados não foi implementado em todos os trabalhos. Exceto os artigos apresentados por Rocha, Costa e Right (2015) e Delir Haghighi et al. (2014), apresentaram o terceiro nível da ciência de situação, os outros três trabalhos tiveram a implementação somente dos dois primeiros níveis.

\section{Modelo SAC}

O modelo SAC - Situation-Aware Care propõe o monitoramento de pacientes, utilizando os sensores e recursos disponíveis nos dispositivos móveis e nos dispositivos vestíveis. Coletando sinais vitais e outros dados, que combinados permitirão ao modelo acompanhar a saúde do paciente, possibilitando assim um maior e melhor conhecimento sobre a saúde do paciente monitorado. O modelo SAC aplica a combinação de lógica Fuzzy e de Redes Neurais Artificiais como método para o raciocínio da ciência de situação. O uso dessas técnicas permite ao modelo identificar e projetar desvios, degradações e problemas na saúde do paciente. O objetivo do modelo é disponibilizar recursos para que hospitais e médicos tenham registros mais apurados sobre o estado de saúde de seus pacientes, permitindo aos mesmos realizarem diagnósticos mais precisos. A Figura 1, expõe a visão geral do modelo.

Foi definida para o modelo SAC uma arquitetura baseada em serviços, conforme pode ser vista pela Figura 2. A escolha por este tipo de arquitetura se deu pelo fato de que a mesma permite a criação de serviços interoperáveis que podem ser facilmente reutilizados e compartilhados entre aplicações [ARSANJANI, 2004]. A seguir serão apresentados cada um dos serviços - Serviços SAC e Serviços Públicos SAC, ambos em nuvem computacional - e clientes disponibilizados pelo modelo SAC, descrevendo sobre as suas funcionalidades e responsabilidades.

\subsection{Clientes SAC4Mobile e SAC4Monitors}

A aplicação SAC4Mobile, disponibilizada para pacientes e médicos através dos dispositivos móveis, tem como responsabilidade prover as seguintes funcionalidades: coleta dos dados pacientes; cadastro usuários; comunicação entre paciente, médico e hospital; monitoramento dos sinais vitais (pacientes); transmitir dados coletados (pacientes); relatar situação atual (pacientes); e solicitar emergência. Este cliente, tem como principal funcionalidade a captura e envio dos dados coletados através dos dispositivos vestíveis e sensores. Após a coleta os dados são enviados para o serviço público SAC, Concentrador de Dados. Os usuários da aplicação SAC4Mobile, possuem acessos específicos, sendo vinculados ao tipo de usuário - paciente ou médico. 


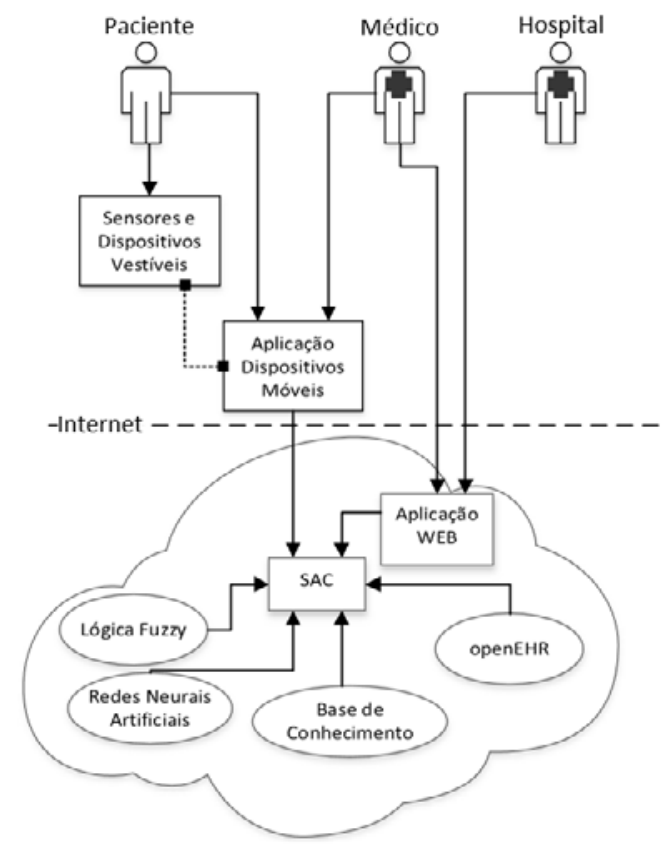

Figura 1. Visão geral modelo SAC

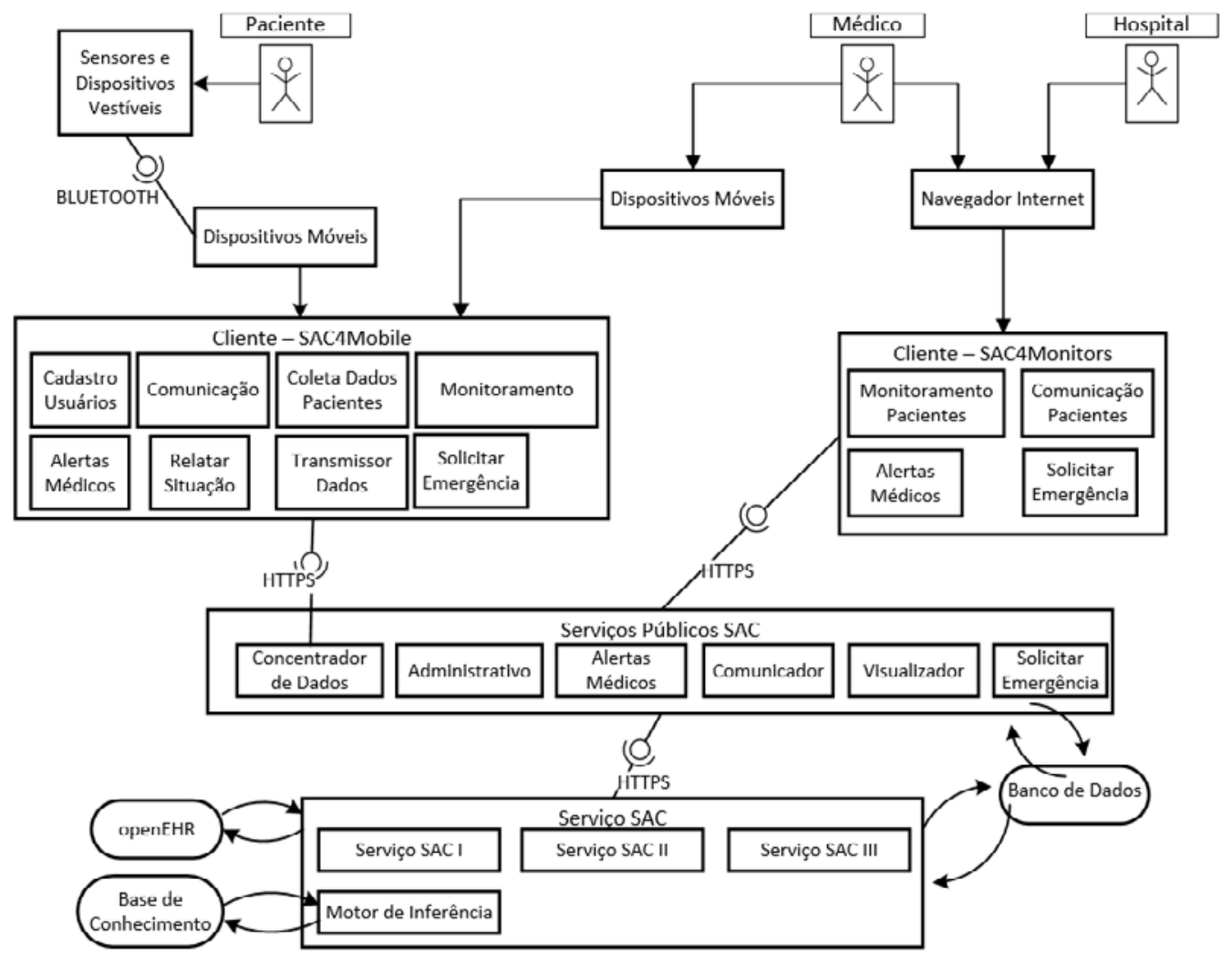

Figura 2. Arquitetura modelo SAC

A aplicação SAC4Monitors, para médicos e hospitais por uma aplicação web, permite o acompanhamento dos pacientes, tendo acesso a visualização dos dados coletados e das inferências obtidas. Permite também a comunicação direta com os pacientes, criação de alertas, que são refletidas em notificações, onde utilizam como 
referência as projeções inferidas pelo serviço SAC III e também o recurso de acionar o resgate médico para um determinado paciente.

\subsection{Serviço SAC I}

O serviço SAC I, em conjunto com a aplicação SAC4Mobile e o componente Concentrador de Dados dos serviços públicos SAC, representam a primeira fase da ciência de situação [ENDSLEY, 1995], a percepção dos elementos. Possui as seguintes funcionalidades: Receptor, aguarda o envio dos dados pelos Serviços Públicos SAC; Validador, valida a integridade dos dados recebidos, desconsiderando ruídos e dados incompletos; Organizador, organiza e padroniza os dados segundo openEHR; Persistência, armazena os dados, tendo como referência a data e hora da coleta de cada dado.

\subsection{Serviço SAC II}

O serviço SAC II, proposto pelo modelo SAC, representa a segunda fase da ciência de situação [ENDSLEY, 1995], compreensão sobre a situação atual. Para atingir esta fase da compreensão da situação atual do paciente, foi escolhida a Lógica Fuzzy. A escolha feita devida ao tratamento de imprecisões, a especificação das regras mais próximo a linguagem natural e simplificação na construção da base de conhecimento [ZADEH, 1996]. O serviço possui as seguintes responsabilidades: Fuzzyficador de Dados, aplica a fuzzyficação nos dados coletados, convertendo-os em expressões linguísticas; Regras de Inferência, constituem a Base de Conhecimento, ou seja, representam o conhecimento do especialista médico. Como resultado, retorna à situação atual do paciente; Defuzzyficador de Resultados, componente realiza a fase final da Lógica Fuzzy, convertendo os resultados fuzzyficados em valores numéricos; Analisador da Situação, analisa os resultados obtidos, e conforme o risco determinado, realiza ação.

\subsection{Serviço SAC III}

A terceira fase da ciência de situação, projeção sobre estados futuros, é aplicada pelo serviço SAC III. O modelo SAC utiliza o conceito de predição de Série de Dados Temporais por Redes Neurais Artificiais para atingir o objetivo proposto pela terceira fase da ciência de situação. O treinamento da Rede Neural se dá com a utilização do histórico do paciente observado, permitindo assim aprender sobre como é o comportamento - tendências, sazonalidades, limites, constâncias, dentre outros padrões - dos dados coletados [MITCHELL, 1997]. Para tal, os seguintes componentes foram especificados: Prognosticador de Dados, responsável pela implementação da Rede Neural, ou seja, realizará a predição da série de dados temporais referente aos dados dos pacientes; Projetor de Saúde, a partir das informações inferidas pelo serviço SAC II, este componente fica responsável por projetar as possíveis degradações na saúde do paciente; Harmonizador de Dados, ao fim das inferências sobre as predições, é realizada uma calibração sobre os dados paciente conforme histórico do mesmo.

\subsection{Serviço Públicos SAC}

Para o acesso aos dados e as informações inferidas, o modelo SAC disponibiliza um pacote de serviços, chamado de Serviços Públicos SAC. Este pacote permite o consumo dos dados e das informações tanto para as aplicações SAC quanto para as aplicações 
terceiras. Este pacote de serviços foi criado para separar as responsabilidades do modelo. Atividades que não são vinculadas diretamente com a ciência de situação foram alocadas para os serviços públicos, deixando assim o serviço SAC responsável apenas por aplicar a ciência de situação. Os recursos disponibilizados pelos serviços públicos SAC seriam: Administrativo, realiza o gerenciamento administrativo do modelo SAC; Comunicador, permite a comunicação direta entre os envolvidos; Concentrador de dados, responsável por receber os dados coletados pela aplicação SAC4Mobile e enviar para o serviço SAC I; Alertas Médicos, envia uma notificação a partir de um alerta gerado por uma das aplicações; Visualizador, permite a visualização dos dados coletados assim como as informações inferidas sobre os pacientes observados; Solicitar Emergência, permite a solicitação de resgate médico em caráter de emergência para o paciente observado.

\section{Avaliação}

A aplicação de cenários, como avaliação de modelos ubíquos vem sendo utilizada pela comunidade científica [QUEVEDO; COSTA; RIGHI; RIGO, 2015] [ROCHA; COSTA; RIGHI, 2015]. É uma ferramenta para visualização de um possível caminho de ação para uma determinada situação, ou seja, ajudam na compreensão do fluxo das aplicações e dos serviços de novas tecnologias imaginadas. Avaliações de cenários também são uma maneira flexível e prática na descoberta de possíveis atitudes e necessidades do usuário. $\mathrm{O}$ valor dos cenários está na representação dos conceitos em descrições mais sólidos para efeitos de análise e apresentação [IKONEN, 2002]. Para o modelo SAC foi realizada a seguinte aplicação de cenário:

"Maria, jovem de 24 anos, mora sozinha e tem o hábito de praticar corridas. Maria trabalha como modelo fotográfica, logo a boa aparência e saúde são seus aliados. Pensando na sua saúde, Maria começou a utilizar a aplicação SAC4Mobile para monitorar o seu dia a dia, em conjunto com um dispositivo vestivel para monitorar a temperatura e uma smartband capaz de coletar a frequência cardíaca. Maria indicou o seu médico particular como referência médica em casos de alerta, dessa forma ele recebe toda e qualquer notificação referente a sua saúde. Durante a prática das corridas, o SAC4Mobile reconhece que ela está se exercitando e por isso considera que a elevação nos batimentos cardíacos está de acordo, assim como o aumento da temperatura. Maria, há dois atrás realizou a sua corrida diária, porém, desta vez foi surpreendida por uma chuva durante a atividade. Após dois dias, o SAC4Mobile apresenta a notificação de um aumento na temperatura de Maria, e durante o dia a mesma reportou estar sentindo dores no corpo e na cabeça. Com essas novas informações enviadas ao serviço SAC, o mesmo indica possível gripe, como projeção para a saúde de Maria. Médico recebe alerta referente a alteração na saúde de Maria, e o mesmo lhe encaminha mais questionamentos sobre o estado atual e também sobre a rotina de Maria durante os dias anteriores, a mesma reporta que foi surpreendida pela chuva. Em posse das inferências apresentadas pelo serviço $S A C$, o médico recomenda a compra de antigripais e repouso, pois, ele tem a mesma opinião que o $S A C$, de que Maria pegou uma gripe. Maria atua conforme recomendado, pois, em quatro dias precisará estar em plena saúde devido a um trabalho fotográfico”.

Tendo em vista o cenário acima apresentado, foi então desenvolvido o protótipo da aplicação SAC4Mobile para a plataforma Android, assim como os Serviços Públicos 
SAC e os Serviços SAC, estes dois hospedados na nuvem, Amazon EC2. O monitoramento dos sinais vitais é realizado através de um dispositivo vestível que monitora a temperatura corporal do paciente - desenvolvido utilizando Arduino Lilypad e sensores de temperatura - e de uma smartband que monitora a frequência cardíaca FitBit Charge HR. Nos protótipos desenvolvidos, o modelo SAC objetivou o menor desconforto ao utilizador da solução. A Figura 3, apresenta o protótipo SAC4Mobile.

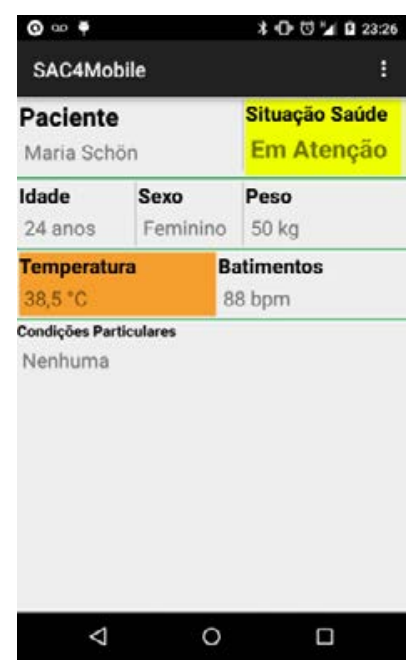

Figura 3. Protótipo SAC4Mobile

Durante as práticas das corridas de Maria, o modelo SAC, compreendeu e projetou, que a situação dela se tratava de uma atividade física e que o aumento na frequência cardíaca e na temperatura estavam de acordo. Entretanto conforme foi apresentado, a paciente realizou atividades em um período chuvoso, durante o monitoramento dos dias seguintes, foram compreendidosm e projetados desvios aumento - na temperatura. Esta projeção aliada aos reportes de situação da paciente, onde a mesma indicava dores no corpo e na cabeça, inferiram na situação de gripe. Ao projetar o desvio na saúde da paciente, é disparada uma notificação tanto para o médico quanto para a paciente monitorada. Com a notificação, permite hospitais e médicos estarem cientes dos desvios e também a realizar alguma forma de intervenção médica. $\mathrm{E}$ com a intervenção realizada pelo médico de Maria, permitiu que a mesma iniciasse o seu tratamento sem mesmo ter realizado uma consulta médica presencial.

Com o desenvolvimento dos protótipos acima descritos e avaliação de cenário, foi então solidificada as expectativas quanto ao modelo SAC e a utilização de ciência de situação para a projeção de futuros estados da saúde. Onde o modelo SAC, através de suas aplicações, monitoramento e serviços, perceberam os desvios nos sinais vitais monitorados, e em conjunto com os reportes da paciente, pôde projetar a tendência dos desvios e tendo por conclusão inferido a situação da paciente. Se apresentando então, como uma solução relevante em relação aos cuidados ubíquos disponibilizando recursos - para médicos e para pacientes - que possibilitam melhores cuidados para com a saúde dos pacientes monitorados e também tomadas de decisões mais acertivas.

\section{Conclusão}

O modelo SAC realiza o monitoramento de sinais vitais de pacientes aliado a aplicação das três fases da ciência de situação sobre dados obtidos. A ciência de situação permite 
ao modelo realizar inferências mais acertivas quanto ao estado ou a situção em que o paciente se encontra, pois, compreende os elementos e suas relações, assim como projeta futuros estados do paciente. Sendo estas inferências, recursos que permitem um maior e um melhor conhecimento sobre a saúde dos pacientes monitorados, possibilitando melhores condições para as tomadas de decisão dos médicos. E o protótipo desenvolvido solidificou as expectativas quanto ao modelo, identificando as situações dos pacientes e realizando as devidas notificações aos envolvidos, permitindo assim que os médicos tenham um tempo de resposta muito rápido em casos de desvios e degradações da saúde. Demonstrando a sua relevância para o domínio dos cuidados ubíquos e também como sendo uma solução de aplicação possível.

Para trabalhos futuros pretende-se incorporar mais dados dos pacientes e consequentemente incluir novas regras de inferências, permitindo assim uma maior abrangência do modelo SAC. Outra perspectiva para o futuro é avaliar o modelo proposto quanto a usabilidade das aplicações e o quanto ao desempenho das inferências, buscando realizar melhorias no modelo SAC.

\section{Agradecimentos}

Os autores gostariam de agradecer o CAPES e ao CNPq pelo apoio a esta pesquisa.

\section{Referências}

ARSANJANI, Ali. Service-oriented modeling and architecture. IBM developer works, p. 1-15, 2004.

DELIR HAGHIGHI, Pari et al. Situation-aware mobile health monitoring. Em: Proceedings of the 11th International Conference on Mobile and Ubiquitous Systems: Computing, Networking and Services. ICST (Institute for Computer Sciences, Social-Informatics and Telecommunications Engineering), 2014. p. 248-256.

DON, S. et al. An awareness approach to analyze ECG streaming data. Journal of medical systems, v. 37, n. 2, p. 1-12, 2013.

ENDSLEY, Mica R. Toward a theory of situation awareness in dynamic systems. Human Factors: The Journal of the Human Factors and Ergonomics Society, v. 37, n. 1, p. 32-64, 1995.

ENDSLEY, Mica R. Situation awareness misconceptions and misunderstandings. Journal of Cognitive Engineering and Decision Making, v. 9, n. 1, p. 4-32, 2015.

FIORATOU, E. et al. Beyond monitoring: distributed situation awareness in anaesthesia. British journal of anaesthesia, v. 105, n. 1, p. 83-90, 2010.

FRIEDEWALD, Michael; RAABE, Oliver. Ubiquitous computing: An overview of technology impacts. Telematics and Informatics, v. 28, n. 2, p. 55-65, 2011.

GELOGO, Yvette E.; KIM, Haeng-Kon. Unified Ubiquitous Healthcare System Architecture with Collaborative Model. International Journal of Multimedia and Ubiquitous Engineering, v. 8, n. 3, p. 239-244, 2013. 
GONZÁLEZ-FERRER, Arturo; PELEG, Mor. Understanding requirements of clinical data standards for developing interoperable knowledge-based DSS: A case study. Computer Standards \& Interfaces, v. 42, p. 125-136, 2015.

IKONEN, Veikko; RENTTO, Katja. Scenario Evaluation for Ubiquitous ComputingStories Come True. Em: Position paper for the Ubicomp 2002 conference workshop Evaluation Methods for Ubiquitous Computing. Goteborg, Sweden. 2002.

MEULEN, Rob van der; WOODS, Viveca. Gartner Says Global Smartphone Sales to Only Grow 7 Per Cent in 2016. 2016. Gartner. Disponível em: <http://www.gartner.com/newsroom/id/3270418>. Acesso em: 11 abril 2016.

MITCHELL, Tom M. Machine Learning. 1997.

PATEL, Mitesh S.; ASCH, David A.; VOLPP, Kevin G. Wearable devices as facilitators, not drivers, of health behavior change. Jama, v. 313, n. 5, p. 459-460, 2015.

PEREIRA, Isaac SA; COSTA, Patrícia Dockhorn; ALMEIDA, João Paulo A. A rulebased platform for situation management. Em: Cognitive Methods in Situation Awareness and Decision Support (CogSIMA), 2013 IEEE International MultiDisciplinary Conference on. IEEE, 2013. p. 83-90.

QUEVEDO, N. M. M. ; COSTA, C. A. da ; RIGHI, R. R. ; RIGO, Sandro J. . Um Modelo Ubíquo de Detecção de Alergia Baseado em Ciência de Situação. Em: 7o Simpósio Brasileiro de Computação Ubíqua e Pervasiva (SBCUP 2015), 2015, Recife. Anais do 7o Simpósio Brasileiro de Computação Ubíqua e Pervasiva (SBCUP 2015). Porto Alegre: SBC, 2015. p. 31-40.

ROCHA, C. C. L. ; COSTA, C. A. da ; RIGHI, R. R. . Um modelo para monitoramento de sinais vitais do coração baseado em ciência da situação e computação ubíqua. Em: 7o Simpósio Brasileiro de Computação Ubíqua e Pervasiva (SBCUP 2015), 2015, Recife. Anais do 7o Simpósio Brasileiro de Computação Ubíqua e Pervasiva (SBCUP 2015). Porto Alegre: SBC, 2015. p. 51-60.

SATYANARAYANAN, Mahadev. Mobile computing: the next decade. Em: Proceedings of the 1 st ACM workshop on mobile cloud computing \& services: social networks and beyond. ACM, 2010. p. 5.

SINGH, Hardeep et al. Exploring situational awareness in diagnostic errors in primary care. BMJ quality \& safety, v. 21, n. 1, p. 30-38, 2012.

WAI, Aung Aung Phyo et al. Situation-aware patient monitoring in and around the bed using multimodal sensing intelligence. Em: Intelligent Environments (IE), 2010 Sixth International Conference on. IEEE, 2010. p. 128-133.

WEISER, Mark. The computer for the 21 st century. Scientific american, v. 265, n. 3, p. 94-104, 1991.

ZADEH, Lotfi A. Fuzzy logic = computing with words. Fuzzy Systems, IEEE Transactions on, v. 4, n. 2, p. 103-111, 1996. 